\title{
Structural analysis based sensors fault detection and isolation of cylindrical lithium-ion batteries in automotive applications
}

\author{
Zhentong Liu ${ }^{\mathrm{a}, \mathrm{b}^{*}}$, Qadeer Ahmed ${ }^{\mathrm{b}}$, Jiyu Zhang ${ }^{\mathrm{b}}$, Giorgio Rizzoni ${ }^{\mathrm{b}}$, Hongwen $\mathrm{He}^{\mathrm{a}}{ }^{\mathrm{*}}$ \\ a National Engineering Laboratory for Electric Vehicles, Beijing Institute of Technology, 5 South Zhongguancun Street, \\ Beijing 100081, China \\ ${ }^{\mathrm{b}}$ Center for Automotive Research, The Ohio State University, 930 Kinnear Rd., Columbus, OH 43212, USA
}

\begin{abstract}
-
The battery sensors fault diagnosis is of great importance to guarantee the battery performance, safety and life as the operations of battery management system (BMS) mainly depend on the embedded current, voltage and temperature sensor measurements. This paper presents a systematic model-based fault diagnosis scheme to detect and isolate the current, voltage and temperature sensor fault. The proposed scheme relies on the sequential residual generation using structural analysis theory and statistical inference residual evaluation. Structural analysis handles the pre-analysis of sensor fault detectability and isolability possibilities without the accurate knowledge of battery parameters, which is useful in the early stages of diagnostic design. It also helps to find the analytical redundancy part of the battery model, from which subsets of equations are extracted and selected to construct diagnostic tests. With the help of state observes and other advanced techniques, these tests are ensured to be efficient by taking care of the inaccurate initial State-of-Charge (SoC) and derivation of variables. The residuals generated from diagnostic tests are further evaluated by a statistical inference method to make a reliable diagnostic decision. Finally, the proposed diagnostic scheme is experimentally validated and some experimental results are presented.
\end{abstract}

Keywords - Lithium-ion battery; Fault detection and isolation; Structural analysis; Statistical inference residual evaluation.

\section{Introduction}

With the development of Electric Vehicles (EVs) in recent years, the lithium-ion batteries, as the energy storage device, are gaining more and more attentions due to its inherent benefits of high energy and power density, low self-discharge rate and long lifespan [1]. To guarantee the battery safety, performance, reliability and life, a welldesigned battery management system (BMS) is required to perform the functions such as thermal management to ensure the batteries work at optimal average temperature and reduced gradient, State-of-Charge (SoC) and State-ofHealth (SoH) estimations, as well as over-current, over-/under-voltage protections [2]-[6]. These critical functions are mainly dependent on the embedded current, voltage and temperature sensor measurements.

\footnotetext{
* Corresponding author

Email address: zhentongliu87@,gmail (Z. Liu)
} 
The sensors in the battery system may present various kinds of faults due to the manufacturing flaws, degradation, and external shock or vibrations [7]. If the current or voltage sensor is faulty, the $\mathrm{SoC}$ and $\mathrm{SoH}$ estimation performance will be degraded. The inaccurate estimated SoC maybe result in the battery suffering from over-charge and/or over-discharge, which would accelerate the process of battery aging [2], [4], [8]. Moreover, the current and voltage protection circuitries may not work properly in the sensor faulty cases. If the temperature sensor cannot work normally, the performance of thermal management will deteriorate, thus decreasing the battery performance and life [9]. Therefore, it is critical to develop a reliable sensor fault detection and isolation (FDI) scheme for the battery system.

Few studies have been conducted regarding the battery fault diagnosis. A bank of reduced order Luenberger observers was used to isolate a single fault for a three-cell battery string, and the major problem of this scheme is that the Luenberger observer cannot achieve a satisfactory performance for system with measurement noise [10]. Nonlinear parity equation based diagnostic scheme was applied to detect the current or voltage sensor fault in the simulation environment, but assuming the temperature sensor must be in no-faulty case [8]. A multiple model-based diagnostic scheme was presented for the lithium-ion battery to detect the over-charge and over-discharge with the use of EKFs, but along with the complexity and difficulty of identifying various models and running a bank of EKFs [11]. From the best of our knowledge, the topic of battery sensor FDI has been rarely addressed.

In this paper, a systematic model-based FDI scheme is proposed for the lithium-ion battery to detect and isolate the current, voltage and temperature sensor fault based on structural analysis theory. The proposed methodology could be applied for any other battery system faults. One advantage of this methodology is that it could pre-analyze the battery sensor fault detectability and isolability without the accurate knowledge of battery parameters, but just depending on the structural information of battery dynamics [12], [13]. This is useful in the early design stages of diagnostic system. A further advantage of the structural analysis method is that it could decompose the coupled battery dynamics that has many internal interactions into smaller subsystems, which allows the design and implementation of efficient and effective diagnostic algorithms. The basic idea is that through analyzing the analytical redundancy part of structural model, structural analysis helps to extract various subsets of battery dynamics to construct diagnostic tests. In each diagnostic test, the unknowns are calculated sequentially using the known variables, and then a redundant equation (or consistency relation) is checked to generate a residual. To address different issues like unknown initial SoC and derivation of battery variables in different diagnostic tests, some efficient and effective diagnostic algorithms are designed based on the state observers and other advanced techniques. Further, in order to make a more accurate diagnostic decision, the residuals are evaluated by a statistical inference method, instead of just using constant alarm thresholds for the residuals. Finally, the effectiveness of the proposed FDI scheme is experimentally validated and some experimental results are presented.

This paper is organized as follows. Section 2 describes the battery modeling. Section 3 gives the background theory of structural analysis based fault diagnosis, while Section 4 presents the proposed FDI scheme. Section 5 
illustrates the experimental design. The experimental diagnostic evaluation and resulting conclusions are given in Section 6 and 7, respectively.

\section{Coupled electro-thermal model}

In this section, a coupled electro-thermal model is described for cylindrical lithium iron phosphate batteries. It is composed of: a second order equivalent circuit model to capture the battery terminal voltage, and a two-state thermal model used to estimate the battery surface and core temperatures. Each individual model is described in the following subsections.

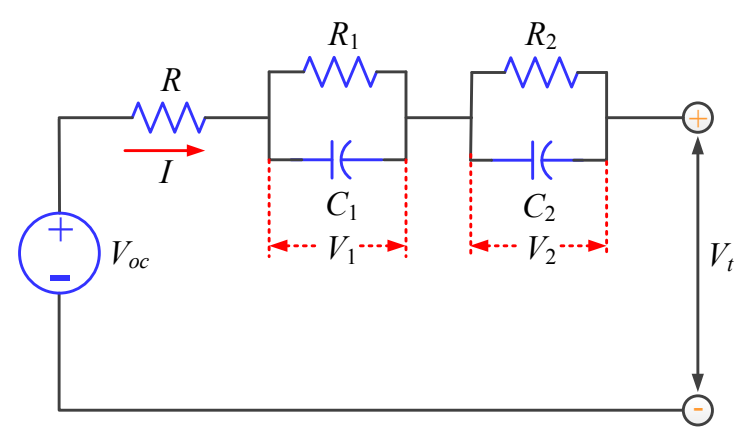

Fig. 1. Schematic of second order equivalent circuit model.

\subsection{Electrical model}

Fig. 1 shows the electrical model, which consists of an open circuit voltage (OCV) $V_{o c}$, an ohmic resistance $R$, and two parallel resistor-capacitors $\left(R_{1}-C_{1}, R_{2}-C_{2}\right)$. The model representation is given as,

$$
\begin{gathered}
\frac{d V_{1}}{d t}=-\frac{V_{1}}{R_{1} C_{1}}+\frac{I}{C_{1}} \\
\frac{d V_{2}}{d t}=-\frac{V_{2}}{R_{2} C_{2}}+\frac{I}{C_{2}} \\
V_{t}=V_{o c}-V_{1}-V_{2}-I \cdot R
\end{gathered}
$$

where $I$ is the current that is positive at discharge and negative at charge, $V_{1}$ is the voltage across capacitor $C_{1}, V_{2}$ is the voltage across the capacitor $C_{2}$, and $V_{t}$ is the terminal voltage.

The battery OCV is usually a nonlinear function of SoC. The SoC is calculated by coulomb counting as,

$$
\frac{d S o C}{d t}=-\frac{I}{C_{b a t}}
$$

where $C_{b a t}$ is the battery available capacity. The equivalent circuit resistances and capacitances depend on the core temperature, SoC and current direction, and the effect of battery aging is not considered in this work. The battery capacity, OCV, parameters $R, R_{1}, C_{1}, R_{2}$, and $C_{2}$ will be identified in the following experimental design section. 


\subsection{Thermal model}

The two-state thermal model, as sketched in Fig. 2, is used to model the radial thermal dynamics of a cylindrical battery (A123 26650) using the core and surface temperatures $T_{c}$ and $T_{s}$, represented as,
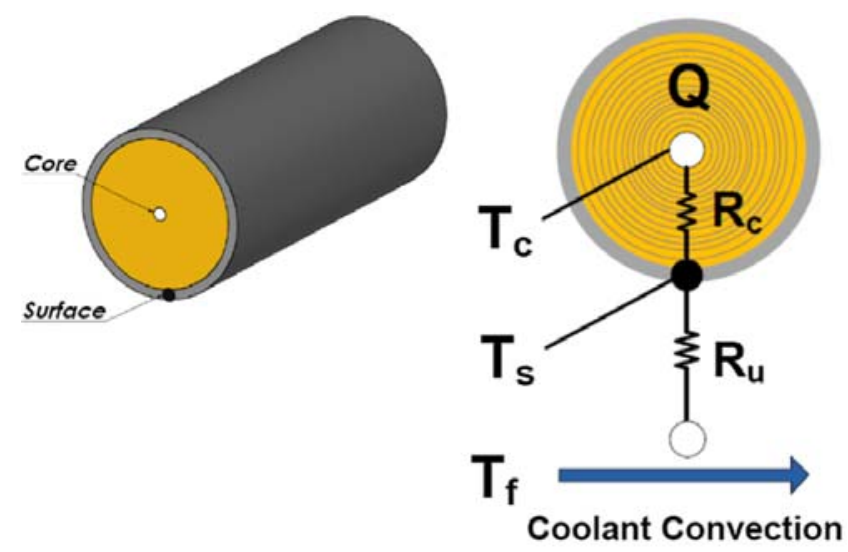

Fig. 2. Schematic of two-state thermal model (Adopted from [14]).

$$
\begin{gathered}
\frac{d T_{c}}{d t}=\frac{T_{s}-T_{c}}{R_{c} C_{c}}+\frac{Q}{C_{c}} \\
\frac{d T_{s}}{d t}=\frac{T_{f}-T_{s}}{R_{u} C_{s}}-\frac{T_{s}-T_{c}}{R_{c} C_{s}} \\
Q=I\left(V_{o c}-V_{t}\right)
\end{gathered}
$$

where $T_{c}$ and $T_{s}$ are the core and surface temperature respectively; $R_{c}, C_{c}, R_{u}$, and $C_{s}$ represents the heat conduction resistance, core heat capacity, convection resistance, and surface heat capacity, respectively; $Q$ is the heat generation due to the joule heating and energy dissipated by electrode over-potentials. As assumed in [14], [15], the coolant flow rate is constant, and the ambient temperature $T_{f}$ is nearly constant in this work.

\subsection{Sensor fault modeling}

For control purposes, the lithium-ion battery system is usually embedded with various kinds of sensors including current, voltage and temperature sensors. Most of the current and voltage sensors used in the battery system of EVs are Hall Effect sensors, which are subject to sensor offset (bias) or scaling fault (gain fault) [2]. These maybe caused by flaws in sensor core (degradation or breakage), temperature variation or mechanical shocks [7]. The temperature sensor is critical for the thermal management, preventing the battery from over-heated or even thermal-runaway. The thermocouples or resistance temperature detectors are widely used, and the fault modes are usually the bias, and scaling [7]. One of the relationships among the sensor measurement, sensor fault and corresponding unknown variable can be represented as [8], [10], [12], [13],

$$
y_{x}=x+f_{x}+\text { noise }
$$


where $x$ is an unknown variable, $y_{x}$ is the signal from sensor installed in the system to measure $x, f_{x}$ is the sensor fault. If there is no sensor fault, the measurement $y_{x}$ would be the actual dynamic value $x$. If there is a sensor fault $f_{x}$ (not zero), the measured value for $x$ would be $x+f_{x}$. Then the system control would be affected by this faulty sensor signal. The sensor fault can be modeled with other relationships [7], but Eq. (8) is considered to model the sensor faults.

\subsection{Full model}

Combing the information of above sub-models, the lithium-ion battery dynamics could be given in Eq. A as follows,

$$
\begin{aligned}
& e_{1}: \frac{d V_{1}}{d t}=-\frac{V_{1}}{R_{1} C_{1}}+\frac{I}{C_{1}}, \quad e_{2}: \frac{d V_{2}}{d t}=-\frac{V_{2}}{R_{2} C_{2}}+\frac{I}{C_{2}} \\
& e_{3}: V_{t}=V_{o c}-V_{1}-V_{2}-I \cdot R, \quad e_{4}: \frac{d S o C}{d t}=-\frac{I}{C_{b a t}} \\
& e_{5}: \frac{d T_{c}}{d t}=\frac{T_{s}-T_{c}}{R_{c} C_{c}}+\frac{Q}{C_{c}}, \quad e_{6}: \frac{d T_{s}}{d t}=\frac{T_{f}-T_{s}}{R_{u} C_{s}}-\frac{T_{s}-T_{c}}{R_{c} C_{s}} \\
& e_{7}: Q=I\left(V_{o c}-V_{t}\right), \quad e_{8}: V_{o c}=f_{1}(S o C) \\
& e_{9}: R=f_{2}\left(T_{c}, S o C, I\right), \quad e_{10}: R_{1}=f_{3}\left(T_{c}, S o C, I\right) \\
& e_{11}: C_{1}=f_{4}\left(T_{c}, S o C, I\right), \quad e_{12}: R_{2}=f_{5}\left(T_{c}, S o C, I\right), \\
& e_{13}: C_{2}=f_{6}\left(T_{c}, S o C, I\right), \quad e_{14}: y_{I}=I+f_{I}, \\
& e_{15}: y_{V_{t}}=V_{t}+f_{V_{t}}, \quad e_{16}: y_{T_{s}}=T_{s}+f_{T_{s}}
\end{aligned}
$$

where $y_{I}, y_{V t}, y_{T s}$ are the measured current, voltage and surface temperature, $f_{I}, f_{V t}, f_{T s}$ are the current, voltage and temperature sensor fault variable. The nonlinear relationship between battery OCV and SoC is represented in $e_{8}$; The nonlinear functions of the electrical parameters $R, R_{1}, C_{1}, R_{2}, C_{2}$ depending on the core temperature, SoC and current direction are given in $e_{9} \sim e_{13}$. The sensor measurements are given in $e_{14} \sim e_{16}$. If there is a sensor fault, the measured unknown variable would affect the system dynamics. Taking the variable $I$ as an example, the $I$ can be measured from signal $y_{I}$, and the $V_{1}$ in $e_{1}$ is then calculated as,

$$
\begin{aligned}
\frac{d V_{1}}{d t} & =-\frac{V_{1}}{R_{1} C_{1}}+\frac{I}{C_{1}} \\
& =-\frac{V_{1}}{R_{1} C_{1}}+\frac{y_{I}}{C_{1}}
\end{aligned}
$$

\section{Background theory of structural analysis based fault diagnosis}

In model based fault diagnosis, the diagnostic information, i.e. residuals, can be generated by checking the analytical redundancy relations (ARR) or consistency relations of the system model. That is, given a system model $M$, system inputs $u$, and observations $y$, ARRs could be found by eliminating all unknown variables sequentially and then leading to a constraint only containing the observed variables, noted $r(u, y)=0$, where $r$ is called the residual of the ARR, and the residuals generated in this way is called sequential residual generation [13], [16]. 
ARRs are violated if the observed system cannot satisfy the system model constraints, i.e. there are faults occurred, then the residual $r(u, y)$ will not be zero any more. It is assumed that $H_{0}$ is the non-faulty hypothesis, and $H_{1}$ is the faulty hypothesis, given as

$$
\begin{cases}r(u, y)=0, & \text { under } H_{0} \\ r(u, y) \neq 0, & \text { under } H_{1}\end{cases}
$$

To detect and isolate different faults, several diagnostic tests (or residual generators) will be required where each test is used to detect some specific faults. Structural analysis based FDI can help to construct each test by using subset of equations extracted from system model. If the system model has more equations than the unknown variables, the model is redundant and contains redundant relations. The redundancy of the system model [17] is defined as,

$$
\varphi(M)=|M|-\left|\operatorname{var}_{X}(M)\right|
$$

where $\varphi(M)$ is the redundancy of system model, $|M|$ is the number of equations included in the system model $M$, and $\left|\operatorname{var}_{x}(M)\right|$ is the number of the unknown variables included in the system model $M$.

\section{Structural analysis based FDI scheme}

Structural analysis based FDI is achieved by the following six steps: 1) Finding the structural over-determined part by analyzing the structural model; 2) Fault detectability and isolability analysis; 3) Minimally structural overdetermined (MSO) sets selection; 4) Diagnostic tests construction based on the selected MSO sets; 5) Residual evaluation; 6) Faults isolation. The schematic of the structural analysis based FDI is shown in Fig. 3.

\subsection{Structural over-determined part}

The structural model can be obtained by analyzing the structural information of the system dynamic, i.e. which variable appears in which equation, and represented in the form of incidence matrix, in which each row of the matrix connects the model equation with the unknown variables, known variables, and faults [18]. The structural model of the battery dynamics, based on Eq. A, is plotted in Fig. 4, in which the set of equations, unknowns, known variables as well as faults are represented as follows,

The set of system equations:

$\left\{e_{1}, e_{2}, \ldots, e_{16}\right\}$,

The set of unknown variables:

$\left\{S o C, I, V_{1}, V_{2}, V_{t}, V_{o c}, R, R_{1}, C_{1}, R_{2}, C_{2}, T_{c}, T_{s}, Q\right\}$,

The set of known variables:

$\left\{y_{I}, y_{V t}, y_{T s}\right\}$,

The set of faults: 
$\left\{f_{I}, f_{V t}, f_{T S}\right\}$.

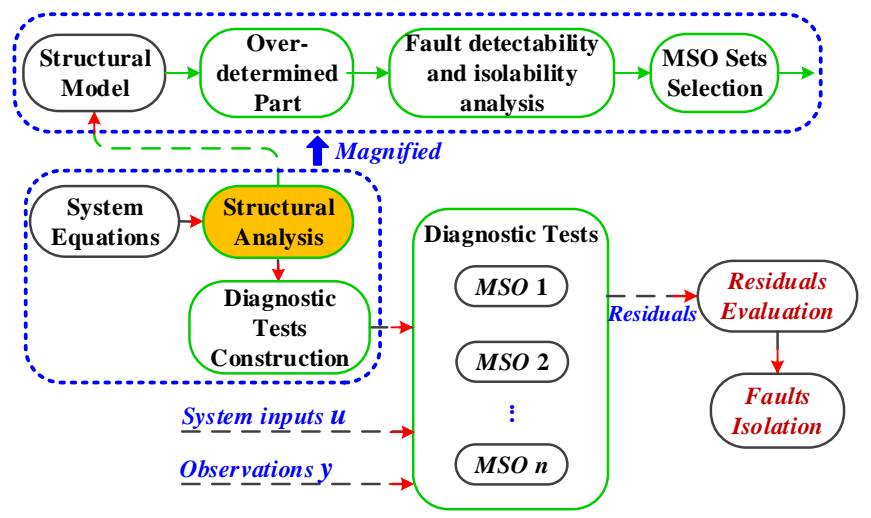

Fig. 3. Schematic of structural analysis based FDI.

The battery model is redundant as there are more equations (16 equations) than unknown variables (14 unknown variables). The structural over-determined part of the system model could be found by analyzing the unknown part of the structural model with Dulmage-Mendelsohn (DM) decomposition [18], which could arrange the rows and columns of the incidence matrix into an upper triangular form, and the unknown part could be decomposed into three parts:

Structural under-determined part $M$; where there are more unknown variables than equations;

Structural just-determined part $M^{0}$, in which the number of equations and unknown variables are equal;

Structural over-determined part $M^{+}$, where there are more equations than the unknown variables.

The decomposed structural model for the lithium-ion battery dynamics is plotted in Fig. 5 .

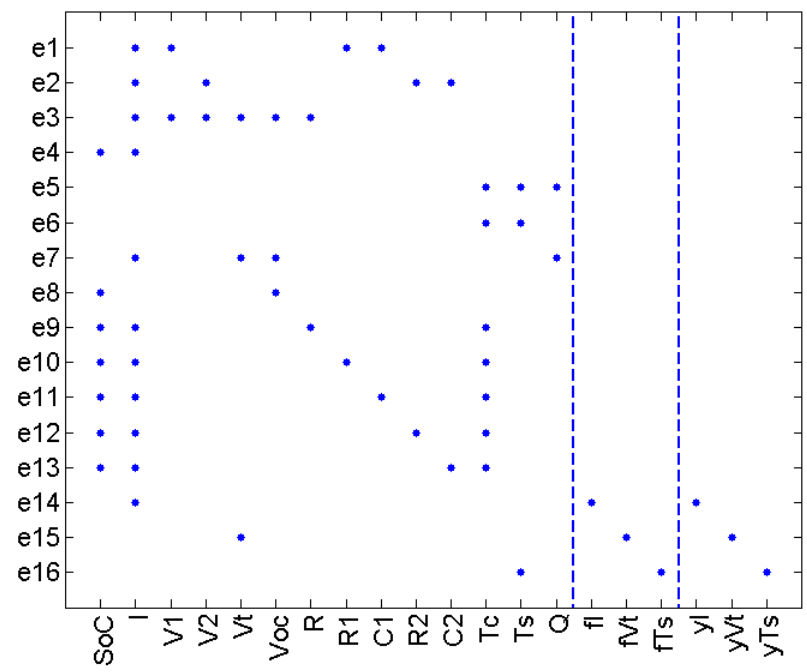

Fig. 4. Structural model of the lithium-ion battery dynamics. 


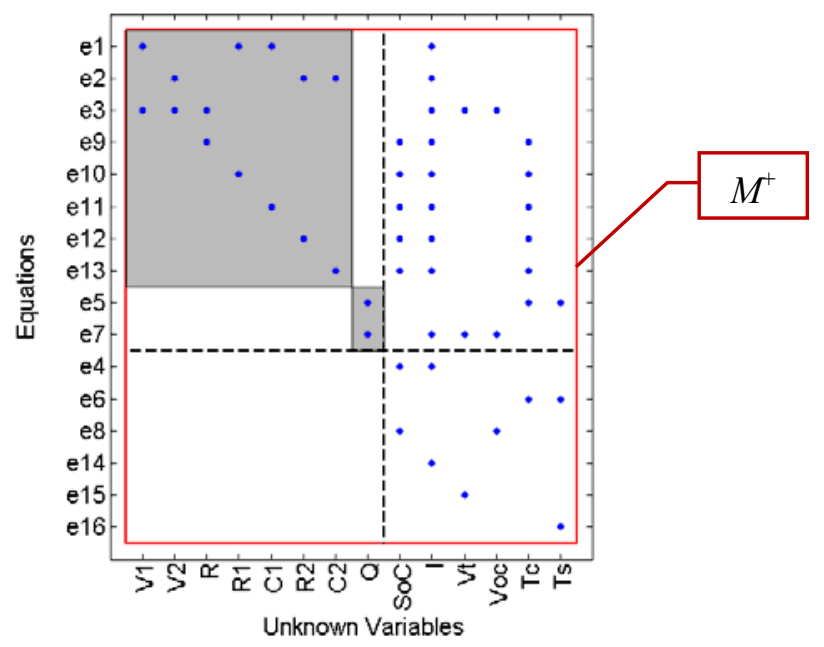

Fig. 5. DM decomposition of the structural model for the lithium-ion battery dynamics.

\subsection{Fault detectability and isolability analysis}

Let $e_{f}$ denotes the equation included in the system model containing fault $f$. It is assumed that only one equation can be violated by each fault. A fault is structurally detectable if the equation with this fault resides in the structural over-determined part [19], defined as,

$$
e_{f} \in M^{+}
$$

It is concluded that in this work all faults can be detected as all the equations with faults $\left(e_{14}, e_{15}\right.$, and $\left.e_{16}\right)$ lies in the structural over-determined part as plotted in Fig. 5.

A fault $f_{i}$ is structurally isolable from fault $f_{j}$ if the fault resides in the structurally over-determined part except the equation the fault $f_{j}$ lies in [19],

$$
e_{f_{i}} \in\left(M / e_{f_{j}}\right)^{+}
$$

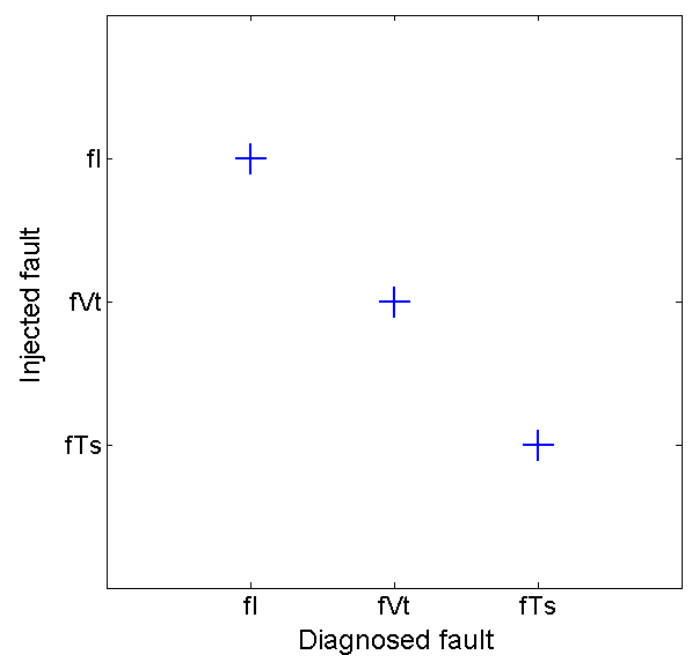

Fig. 6. Fault isolability matrix for the lithium-ion battery dynamics. 
The fault isolability matrix based on the above definition could be obtained as shown in Fig. 6 . It is clear that all the faults can be isolated from each other uniquely.

\subsection{MSO sets selection}

A diagnostic test can be constructed by using a MSO set, in which the redundancy is one. The principle of finding a MSO set is to use as fewer equations as possible, while maintain the redundancy to generate a residual with the redundant relation [17], [20], [21]. The advantage of using MSO set is able to decompose the complex system model into simpler and smaller manageable sub-models, in which the diagnostic algorithm can be easily designed. In each MSO set, a redundant equation could be found to generate a residual by eliminating unknown variables sequentially using the rest of equations. The algorithm in [17] can efficiently find all possible MSO sets, from which the designers can choose the proper number of MSO sets, depending on the system diagnostic requirements.

In addition, the selection of MSO sets needs to consider the integral and derivative causality as there may contain the differential equations in the system model [22]. Taking $e_{1}$ in Eq. A as an example, the integral causality is used when $V_{1}$ is calculated from $I$, but the initial condition of $V_{1}$ needs to be known. On the other hand, if $I$ is calculated from $V_{1}$, the derivative causality is applied, and this may cause the instability.

Each MSO set is sensitive to some certain faults as the inputs to each MSO set may include different inputs $u$ and sensor measurements $y$, and hence faults in any of these sensors can be detected by this MSO set. However, all faults can be efficiently isolated by using various MSO sets as different faults affect each MSO set differently. It is assumed that the simultaneous appearance of different faults is excluded. It is reasonable because the probability of two sensors being simultaneous faulty is quite low.

In this work, there are nine MSO sets found using the algorithm in [17]. In order to isolate the current, voltage and temperature sensor faults, only two MSO sets are needed [23], and the selected MSO sets are shown in Table 1.

Table 1 Selected MSO sets.

\begin{tabular}{cc}
\hline \hline & Contained equations \\
\hline MSO1 & $\left\{e_{1} \sim e_{15}\right\}$ \\
MSO2 & $\left\{e_{1} \sim e_{13}, e_{15}, e_{16}\right\}$ \\
\hline \hline
\end{tabular}

\subsection{Diagnostic tests construction}

Based on the selected MSO sets, the diagnostic tests are constructed with the design of some proper and efficient diagnostic algorithms. The equations used to construct each test are given in Table 1.

\subsubsection{Test 1-MSO1}


MSO1 can be used to test the current or voltage sensor fault as the sensor measurements are contained as given in Table 1. Theoretically, each of the equations in this MSO could be the consistency relation. However, since this MSO is used to test the current or voltage sensor fault, it is natural to choose an equation both having the current and voltage variables, that is $e_{3}$. Since the current $I$ and voltage $V_{t}$ are measured from $e_{14}$ and $e_{15}\left(y_{I}\right.$ and $\left.y_{V t}\right)$, hence the residual $r_{1}$ can be expressed as,

$$
\begin{aligned}
r_{1} & =V_{t}-\left(V_{o c}-V_{1}-V_{2}-I \cdot R\right) \\
& =y_{V_{t}}-\left(V_{o c}-V_{1}-V_{2}-y_{I} \cdot R\right)
\end{aligned}
$$

The other unknown variables $V_{o c}, V_{1}, V_{2}$ and $R$ can be calculated sequentially from the rest of equations in MSO1. The computation sequence (CS) for the unknown variables in Eq. (14) is given as,

$$
C S_{M S O 1}=\left\{\begin{array}{l}
\left(I, e_{14}\right),\left(V_{t}, e_{15}\right),\left(S o C, e_{4}\right),\left(V_{o c}, e_{8}\right), \\
\left(Q, e_{7}\right),\left(\left\langle T_{c}, T_{s}\right\rangle,\left\langle e_{5}, e_{6}\right\rangle\right), \\
\left(\left\langle R, R_{1}, C_{1}, R_{2}, \mathrm{C}_{2}\right\rangle,\left\langle e_{9} \sim e_{13}\right\rangle\right), \\
\left(V_{1}, e_{1}\right),\left(V_{2}, e_{2}\right),
\end{array}\right\}
$$

Taking $e_{4}$ to calculate $\mathrm{SoC}$ as an example,

$$
\begin{aligned}
\frac{d S o C}{d t} & =-\frac{I}{C_{b a t}} \\
& =-\frac{y_{I}}{C_{b a t}}
\end{aligned}
$$

From $e_{4}$, the SoC is calculated from the measured current $y_{I}$ using the integral causality. This requires the initial $\mathrm{SoC}$ is known. Since there is no current flow at the starting point, the measured battery voltage would be the OCV, and the initial SoC can be determined from the OCV-SoC table [24]. The additional better way to address the issue of unknown initial SoC, is using the closed-loop observers. Extended Kalman filter (EKF) has been extensively used to estimate the SoC with the inherent benefits of good performance and easy implementable [2], [4], and therefore it is applied. The battery states are defined as $x=\left[V_{1}, V_{2}, S o C\right]^{\mathrm{T}}$, and the state-space and measurement equation in discrete-time can be expressed as,

$$
\begin{gathered}
x_{k}=A_{k-1} \cdot x_{k-1}+B_{k-1} \cdot I_{k-1} \\
=\left[\begin{array}{ccc}
-\frac{\Delta t}{R_{1} C_{1}}+1 & 0 & 0 \\
0 & -\frac{\Delta t}{R_{2} C_{2}}+1 & 0 \\
0 & 0 & 1
\end{array}\right] \cdot x_{k-1}+\left[\begin{array}{c}
\frac{\Delta t}{C_{1}} \\
\frac{\Delta t}{C_{2}} \\
\frac{-\Delta t}{C_{b a t}}
\end{array}\right] \cdot I_{k-1} \\
y_{k}=C_{k} \cdot x_{k}+D_{k} \cdot I_{k} \\
=\left[\begin{array}{lll}
-1 & -1 & \frac{\partial V_{o c}}{\partial S o C}
\end{array}\right] \cdot x_{k}-R \cdot I_{k}
\end{gathered}
$$


where $x_{k}$ and $x_{k-1}$ are the state variables at the time step $k$ and $k$-1, respectively. $y_{k}$ is the terminal voltage, $\Delta t$ is the time interval. The EKF estimation sequence including the time update and measurement update are given as [25],

$$
\left\{\begin{array}{l}
\hat{x}_{k}^{-}=A_{k-1} \cdot \hat{x}_{k-1}+B_{k-1} \cdot I_{k-1} \\
P_{k}^{-}=A_{k-1} P_{k-1} A_{k-1}{ }^{T}+C o v_{-} w \\
G_{k}=P_{k}^{-} C_{k}^{T}\left[C_{k} P_{k}^{-} C_{k}^{T}+C o v_{-} v\right]^{-1} \\
\hat{x}_{k}=\hat{x}_{k}^{-}+G_{k}\left[y_{V_{t}, k}-\left(C_{k} \hat{x}_{k}^{-}+D_{k} I_{k}\right)\right] \\
P_{k}=\left(I-G_{k} C_{k}\right) P_{k}^{-}
\end{array}\right.
$$

where $\hat{x}_{k}^{-}$and $P_{k}^{-}$are the priori state and error covariance estimates at step $k$, respectively; $\hat{x}_{k}$ and $P_{k}$ represent the posteriori state and error covariance estimates respectively. $G_{k}$ is the Kalman gain, and $y_{V t, k}$ is the measured battery terminal voltage. Cov_w and $C o v_{-} v$ are the process and measurement noise covariance respectively. The processes of sequential calculation and updating parameters are illustrated in Fig. 7. First, the SoC is estimated using EKF based on the measured current $y_{I}$, voltage $y_{V t}$, and electrical parameters $\left(V_{o c}, R, R_{1}, C_{1}, R_{2}, C_{2}\right)$. The $V_{o c}$ is updated based on the estimated SoC. The voltage difference between $V_{o c}$ and $y_{V t}$, along with current $y_{I}$, are used to calculate $Q$. Then the temperature $T_{c}$ and $T_{s}$ are calculated based on $Q$ and ambient temperature $T_{f}$. The core temperature $T_{c}$, along with $\mathrm{SoC}$ and measured current $y_{1}$, are used to update the parameters $R, R_{1}, C_{1}, R_{2}$, and $C_{2}$. Finally, the voltage difference between the measured and estimated terminal voltage is calculated, and the new residual $r_{1}$ could be rewritten into,

$$
r_{1, k}=y_{V_{t}, k}-\hat{V}_{t, k}=y_{V_{t}, k}-\left(C_{k} \hat{x}_{k}+D_{k} I_{k}\right)
$$

where $\hat{V}_{t, k}$ represent the estimated battery terminal voltage. The integral causality is applied when calculating $T_{c}$ and $T_{s}$ from $e_{5}$ and $e_{6}$. The initial value of $T_{c}$ and $T_{s}$ are the ambient temperature $T_{f}$.

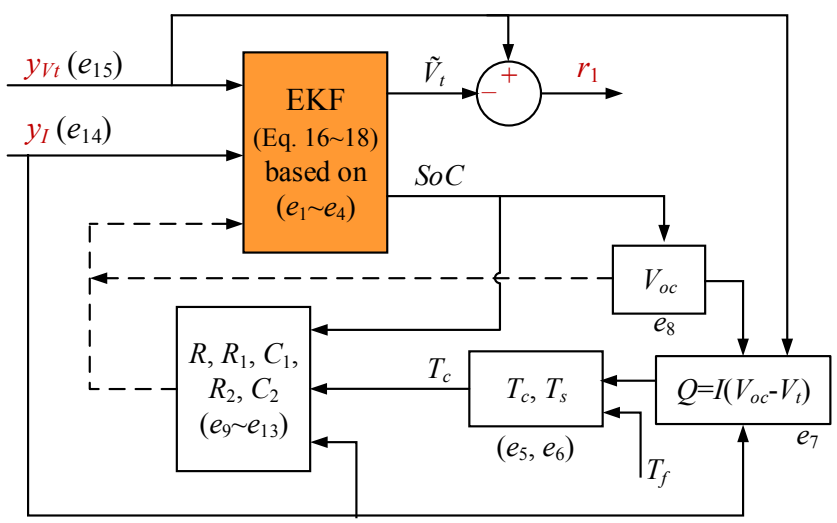

Fig. 7. The processes of sequential calculation and updating parameters for MSO1.

\subsubsection{Test 2-MSO2}


Since this MSO contains the voltage and surface temperature measurements, it can be used to test the voltage sensor or surface temperature sensor fault. It is natural to choose the $e_{5}$ as the consistency relation as both the voltage and surface temperature variables are contained. However, the unknown $T_{c}$ in $e_{5}$ needs to be calculated from $e_{6}$ using the derivative of $T_{s}$. Then the $e_{5}$ is used to generate a residual by differentiating $T_{c}$. It can be found that the derivation of variable is used twice if using $e_{5}$ as the consistency relation, and this may cause divergence [20]. Considering the test stability and sequential calculation for the unknown variable, the $e_{6}$ is selected as the consistency relation, and the residual $r_{2}$ is calculated as,

$$
\begin{aligned}
r_{2} & =\frac{d T_{s}}{d t}-\left(\frac{T_{f}-T_{s}}{R_{u} C_{s}}-\frac{T_{s}-T_{c}}{R_{c} C_{s}}\right) \\
& =\dot{y}_{T_{s}}-\left(\frac{T_{f}-y_{T_{s}}}{R_{u} C_{s}}-\frac{y_{T_{s}}-T_{c}}{R_{c} C_{s}}\right)
\end{aligned}
$$

To avoid the differentiated variable $y_{T s}$, a new state variable $x$ and tuning parameter $\beta$ are introduced. The transformation of Eq. (21) and new residual are expressed as,

$$
\begin{gathered}
\dot{x}=-\beta\left(x+y_{T_{s}}\right)-\left(\frac{T_{f}-y_{T_{s}}}{R_{u} C_{s}}-\frac{y_{T_{s}}-T_{c}}{R_{c} C_{s}}\right) \\
\tilde{r}_{2}=x+y_{T_{s}}
\end{gathered}
$$

Eq. (22) and (23) can be transformed into,

$$
\dot{\tilde{r}}_{2}=-\beta \tilde{r}_{2}+\dot{y}_{T_{s}}-\left(\frac{T_{f}-y_{T_{s}}}{R_{u} C_{s}}-\frac{y_{T_{s}}-T_{c}}{R_{c} C_{s}}\right)
$$

where $\beta>0$ to ensure the stability, $\lim _{t \rightarrow \infty} \tilde{r}_{2}=0$.

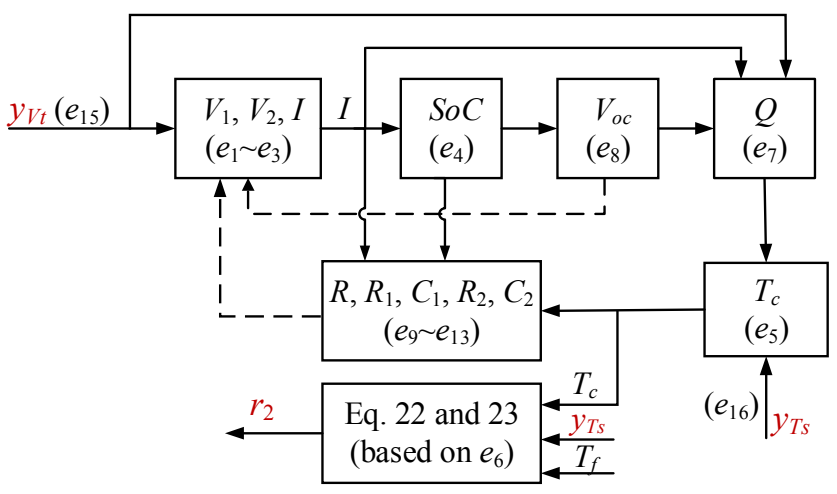

Fig. 8. The processes of sequential calculation for unknown variables and updating parameters for MSO2.

In Eq. (22), the initial value of new state $x$ is the starting value of measured signal $y_{T s}$. The unknown variable $T_{c}$ could be calculated from the rest of equations in MSO2 in a sequence. The processes of sequential calculation for 
unknown variables and updating parameters for MSO2 is illustrated in Fig. 8. Similarly, in $e_{4}$, the initial SoC can be determined from the OCV-SoC table using the measured voltage signal $y_{V t}$ at the starting point [24].

\subsection{Residual evaluation}

To make an accurate diagnostic decision, the statistical cumulative sum (CUSUM) test is applied [26], instead of the conventional residual evaluation approaches like applying a fixed threshold for the residual. The idea of CUSUM algorithm is based on the repeated use of Sequential Probability Ratio Test (SPRT). The SPRT, given by detection theory, is most suitable for testing two hypotheses (no-fault hypothesis $H_{0}$ and faulty hypothesis $H_{1}$ ) against each other to decide which of them describe the observed data (residual). The SPRT is applied by comparing the log likelihood ratio of the observed residual $z(k)$,

$$
s(z(k))=\ln \left(\frac{p\left(z(k), H_{1}\right)}{p\left(z(k), H_{0}\right)}\right)
$$

where $p\left(z(k), H_{1}\right)$ denotes the probability density function (PDF) of observed residual $z(k)$ under the faulty hypothesis $H_{1}, p\left(z(k), H_{0}\right)$ denotes the PDF of observed residual $z(k)$ under the no-faulty hypothesis $H_{0}$.

The decision rule is defined as,

$$
\operatorname{dec}=\left\{\begin{array}{lll}
0 & \text { if } & s_{k} \leq \varepsilon_{1} \\
1 & \text { if } & s_{k} \geq \varepsilon_{2}
\end{array}\right.
$$

It is proved that the optimal lower threshold $\varepsilon_{1}$ should be zero. The idea of CUSUM algorithm is to restart the SPRT algorithm as long as the previously decision $d e c=0$, and the resulting decision rule of the repeated SPRT can be expressed recursively as,

$$
g_{k}=\left\{\begin{array}{ccc}
g_{k-1}+s(z(k)) & \text { if } & g_{k}+s(z(k))>0 \\
0 & \text { if } & g_{k}+s(z(k))<0
\end{array}\right.
$$

The value gk will be increasing once the fault occurs, and the fault detection decision can be defined as,

$$
d=\left\{\begin{array}{lll}
1 & g_{k} \geq J & H_{1} \text { accepted } \\
0 & g_{k}<J & H_{0} \text { accepted }
\end{array}\right.
$$

where $J$ is pre-defined alarm threshold calibrated through experiments, and the alarm signal $d=1$ means that a fault is detected.

\subsection{Fault isolation}

The residuals characteristics for MSO1 and MSO2 are summarized in Table 2, where " $\mathrm{x}$ " is defined as this residual may be used to detect the faults in the corresponding columns. The residual $r_{1}$ is sensitive to the current or voltage sensor fault, while $r_{2}$ is sensitive to the voltage or surface temperature sensor fault. The fault isolation could be explained as following, if only residual $r_{1}$ responds, i.e. only $d_{1}=1$, the column with $f_{I}$ is considered, and hence 
the fault $f_{I}$ is identified. If both residual $r_{1}$ and $r_{2}$ respond, the fault $f_{V t}$ would be located. Similarly, the fault $f_{T s}$ is isolated if only residual $r_{2}$ responds.

Table 2 Residuals characteristics for MSO1 and MSO2.

\begin{tabular}{cccc}
\hline \hline & $f_{I}$ & $f_{V t}$ & $f_{T s}$ \\
\hline$r_{1}$ & $\mathrm{x}$ & $\mathrm{x}$ & \\
$r_{2}$ & & $\mathrm{x}$ & $\mathrm{x}$ \\
\hline \hline
\end{tabular}

\section{Experimental design}

The aim of this section is to identify the battery electrical/thermal model through experimental tests before implementing the diagnostic scheme. Experiments have been conducted on a cylindrical A123 26650 lithium iron phosphate battery with rated capacity of $2.3 \mathrm{Ah}$ and nominal voltage of $3.3 \mathrm{~V}$. The experimental setup is composed of an $800 \mathrm{~W}$ programmable electric load, a $1.2 \mathrm{~kW}$ programmable power supply, a data acquisition unit, a Peltier junctions and associated controllers to provide a controlled thermal environment for the tested battery for temperatures ranging from $-25^{\circ} \mathrm{C}$ to $60^{\circ} \mathrm{C}$, and a computer used for controlling the electric load and supply and also storing the data. A low pass filter function was incorporated into the measurement units to eliminate the large noise, and the current, voltage and temperature data are collected at a sampling frequency of $10 \mathrm{~Hz}$. The experimental setup and sensors were calibrated to guarantee the best possible true measurements.

The battery SoC during the test is calculated by current integration, and thus the initial SoC must be assessed carefully. To guarantee the initial $\mathrm{SoC}$ is always known, prior to each test, the battery is charged to $100 \%$. For the charge process, it is only valid at room temperature specified by the manufacturer as the battery accepts charge differently at different temperatures. Therefore, to keep the uniformity of datasets, the battery is put at the room temperature for three hours before charging the battery to $100 \%$. After the charging is completed, the battery is put at the desired testing temperature for three hours to ensure the battery is at rest before any test is run.

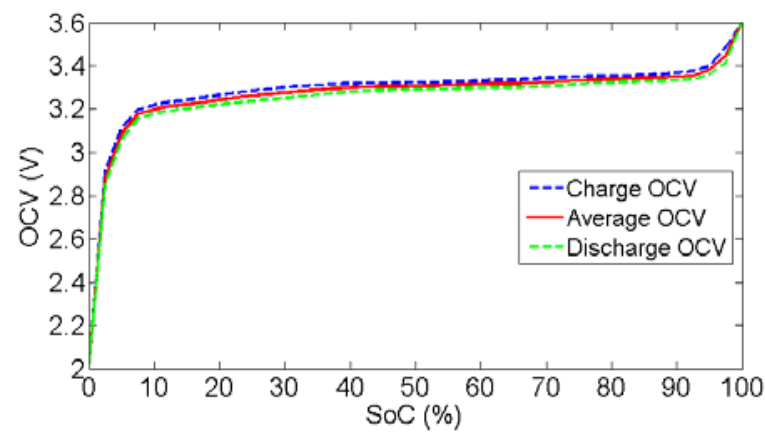

Fig. 9. The tested battery open circuit voltage.

\subsection{Model parameters identification}


The tested available capacity $C_{b a t}$ is $2.16 \mathrm{Ah}$. The OCV test was conducted to calibrate the nonlinear relationship between the $\mathrm{OCV}$ and SoC, and the OCV during charging and discharging are plotted in Fig. 9. The upper and lower voltage limit is $3.6 \mathrm{~V}$ and $2.6 \mathrm{~V}$ respectively. Due to the hysteresis effect of $\mathrm{LiFePO}_{4}$ cell chemistry, there is a voltage difference between the charge and discharge curve. In this work, the hysteresis phenomenon is neglected [14], and the average OCV is taken.

The equivalent circuit parameters are identified based on the hybrid pulse current (HPC) test. This test was conducted at different temperatures $\left(5^{\circ} \mathrm{C}, 20^{\circ} \mathrm{C}, 35^{\circ} \mathrm{C}\right.$ and $\left.55^{\circ} \mathrm{C}\right)$. During each HPC test, to ensure very tight tolerance on the battery temperature under any current profiles, the tested battery is fixed in a specially machined aluminum plates with a large thermal mass, sandwiched between the Peltier junctions. For example, Fig. 10 plots the typical period of the pulse current test at $\mathrm{SoC} 90 \%$ at the temperature $20^{\circ} \mathrm{C}$. There are different current pulses applied ranging from 1C to 4C as shown in Fig. 10 (a), and each pulse will be used to identify the parameters. The ohmic resistance $R$ is calculated by using the instantaneous voltage change $\triangle U$ as [27],

$$
R=\frac{\Delta U}{I_{p u l s e}}
$$

where $I_{p u l s e}$ is the applied current during the pulse, and $\triangle U$ is the instantaneous voltage change as shown in Fig. 10 (d). The other resistance $R_{i}$ and capacitance $C_{i}(i=1,2)$ are calculated by using the relaxation voltage $U_{p u l s e}$ through the least square fitting technique as [27],

$$
\begin{gathered}
U_{\text {pulse }}=\Delta U+\sum_{i=1}^{2} I_{\text {pulse }} R_{i}\left(1-e^{-\frac{t_{\text {pulse }}}{R_{i} C_{i}}}\right) \\
J=\min _{R_{i}, C_{i}} \sum_{k}\left(U_{\text {pulse }}(k)-U_{\text {pulse_data }}(k)\right)^{2}
\end{gathered}
$$

where $t_{p u l s e}$ is the time duration during the relaxation, and $U_{\text {pulse_data }}(k)$ is the measured relaxation voltage at the time instant $k$. Similarly, the parameters could be identified at other SoC points. The detail processes of capacity, OCV and hybrid pulse tests can be found in [28]. Finally, the identified parameters $R, R_{1}, C_{1}, R_{2}$, and $C_{2}$ at discharge are plotted in Fig. 11. It can be found from Fig. 11 (a) that $R$ has a little dependence on SoC, and strong dependence on temperature. The resistance $R$ decreases as the temperature increases. The dependency of $R$ on the temperature can be fitted by an Arrhenius-like function as [14],

$$
R=R_{r e f} \exp \left(\frac{T_{r e f R}}{T_{m}-T_{\text {shiffR }}}\right)
$$

It can be seen from Fig. 11 (b) and (c) that similarly to $R$, the resistance $R_{1}$ and $R_{2}$ decreases as the temperature rises. But $R_{1}$ and $R_{2}$ have a more notable dependency on battery SoC. The dependency of resistance $R_{1}$ and $R_{2}$ on $\mathrm{SoC}$ is fitted by a second order polynomial, and an Arrhenius-like function is used to fit the temperature dependency [14], 


$$
R_{i}=\left(R_{i, 0}+R_{i, 1}(S o C)+R_{i, 2}(S o C)^{2}\right) \exp \left(\frac{T_{r e f R_{i}}}{T_{m}-T_{\text {shiffR }}}\right)
$$

For capacitance $C_{1}$ and $C_{2}$ as shown in Fig.11 (d) and (e), it can be seen that these values are also dependent on the temperature and SoC. But the difference from the resistance $R_{1}$ and $R_{2}$ is that these values increase with the temperature. An exponential function is used to fit the temperature dependency of $C_{1}$ and $C_{2}$, and a second order polynomial for the SoC dependency,

$$
C_{i}=\left(C_{i, 0}+C_{i, 1}(S o C)+C_{i, 2}(S o C)^{2}\right) \exp \left(T_{r e f C_{i}} T_{m}\right)
$$

where $i=1,2 ; T_{m}$ represents the current temperature; $T_{\text {refR }}, T_{\text {shiffR }}, T_{\text {refRi }}, T_{\text {shiffRi}}$, and $T_{\text {refCi } i}$ are the fitted parameters for the temperature dependency; $R_{i, 0}, R_{i, 1}, R_{i, 2}, C_{i, 0}, C_{i, 1}$, and $C_{i, 2}$ are for the SoC dependency. The values of these fitted parameters are summarized in Table 3.

(a)
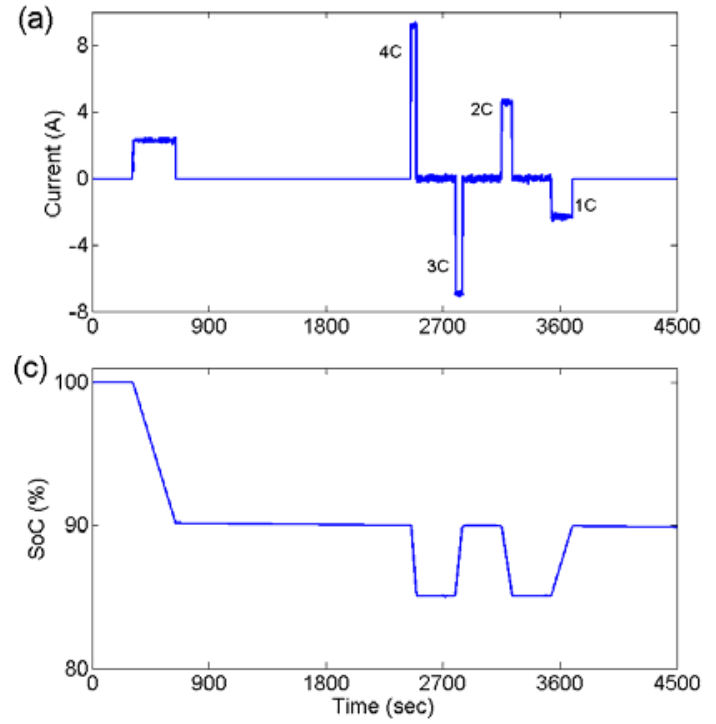

(b)

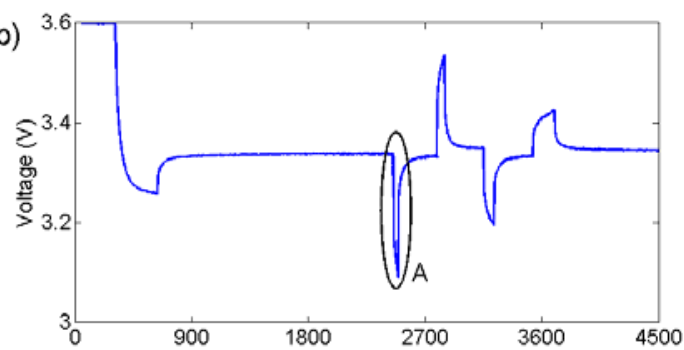

(d)

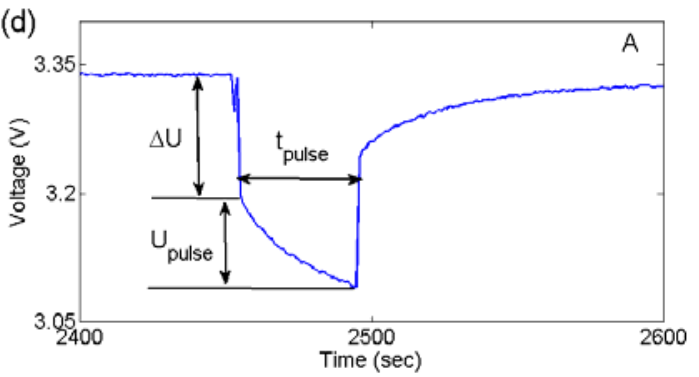

Fig. 10. Typical period of pulse current test at $\mathrm{SoC} 90 \%$ under the temperature $20{ }^{\circ} \mathrm{C}$. (a) Current pulses from $1 \mathrm{C}$ to 4C; (b) Corresponding voltage; (c) SoC trajectory; (d) Amplified voltage when the 4C current pulse is applied. 
(a)
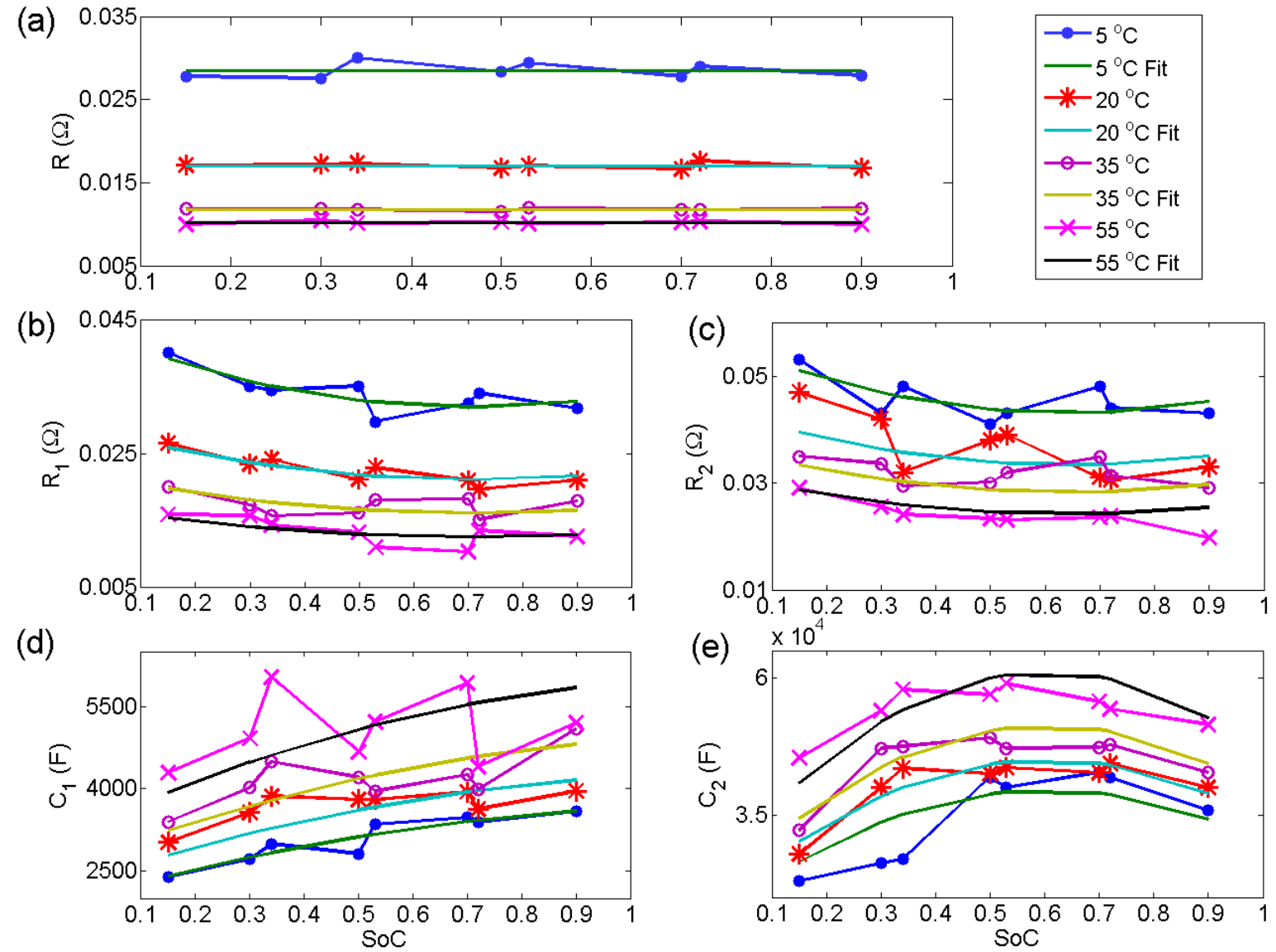

Fig. 11. Equivalent circuit parameters identification results at discharge. (a) Identified $R$; (b) Identified $R_{1}$; (c) Identified $R_{2}$; (d) Identified $C_{1}$; (e) Identified $C_{2}$.

Table 3 Parameter values of the fitting functions for $R, R_{i}$ and $C_{i}$

\begin{tabular}{ccccccc}
\hline \hline$R_{\text {ref }}$ & $T_{\text {refR }}$ & $T_{\text {shiftR }}$ & $R_{1,0}$ & $R_{1,1}$ & $R_{1,2}$ & $T_{\text {refR } 1}$ \\
\hline 0.005 & 61.48 & -30.63 & 0.0055 & -0.0041 & 0.0029 & 123.6 \\
\hline$T_{\text {shiftR } 1}$ & $R_{2,0}$ & $R_{2,1}$ & $R_{2,2}$ & $T_{\text {refR2 }}$ & $T_{\text {shiftR2 }}$ & $C_{1,0}$ \\
\hline-54.79 & 0.0159 & -0.0118 & 0.0092 & 76.07 & -54.79 & 1928.8 \\
\hline$C_{1,1}$ & $C_{1,2}$ & $T_{\text {refC1 }}$ & $C_{2,0}$ & $C_{2,1}$ & $C_{2,2}$ & $T_{\text {refC2 }}$ \\
\hline 2636.5 & -1091.6 & 0.0097 & 15770 & 72813 & -59994 & 0.0087 \\
\hline \hline
\end{tabular}

The adopted thermal model parameters have been accurately calibrated in [14] (the same battery is used in this work), and summarized in Table 4. The thermal model has been validated over a broad range of loading conditions. More details about the thermal experimental design, parameterization, and validations can be found in [14], [15].

Table 4 Two-state thermal model parameters.

$\begin{array}{llll}R_{c}(\mathrm{~K} / \mathrm{W}) & R u(\mathrm{~K} / \mathrm{W}) & C_{c}(\mathrm{~J} / \mathrm{K}) & C s(\mathrm{~J} / \mathrm{K})\end{array}$




\subsection{Model validation by driving cycles}

The scaled Dynamic Stress Test (DST) cycle was used for the battery to simulate the actual driving cycle of electric vehicles [28]. Before this driving cycle test, the battery was put at $20^{\circ} \mathrm{C}$ of a thermal chamber for more than three hours, and the initial SoC is set to $100 \%$. Note that the thermal chamber is used to control the ambient temperature because of its programmability, instead of the Peltier junctions. The ambient temperature $T_{f}$ is controlled at $20^{\circ} \mathrm{C}$. Seven DST cycles were performed to validate the model with identified parameters. The current, SoC, voltage and temperature variations are plotted in Fig. 12 and 13. The SoC varies between 100\% and $20 \%$, while the temperature fluctuates between $20^{\circ} \mathrm{C}$ and $29^{\circ} \mathrm{C}$. The root mean squares errors (RMSE) for the voltage and surface temperature are around $28.6 \mathrm{mV}$ and $0.39^{\circ} \mathrm{C}$, which verify that the identified parameters are accurate and this coupled electro-thermal model is reasonable.
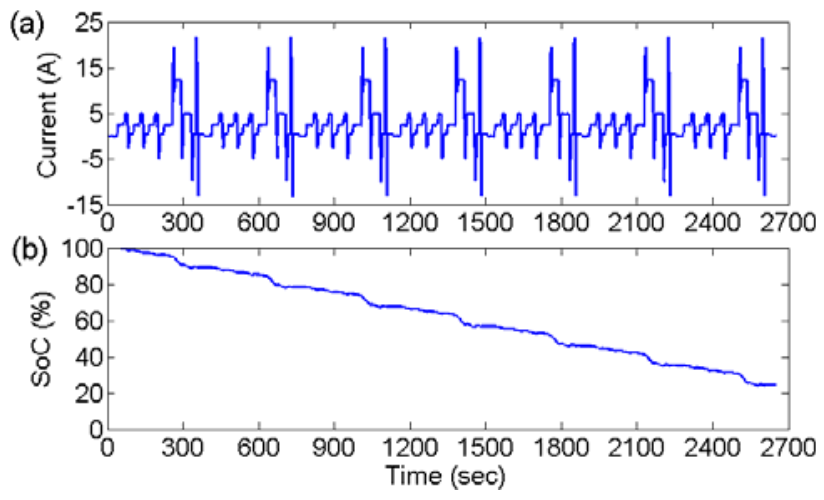

Fig. 12. Current profile and SoC variations of the DST cycles. (a) Input current; (b) SoC trajectory.
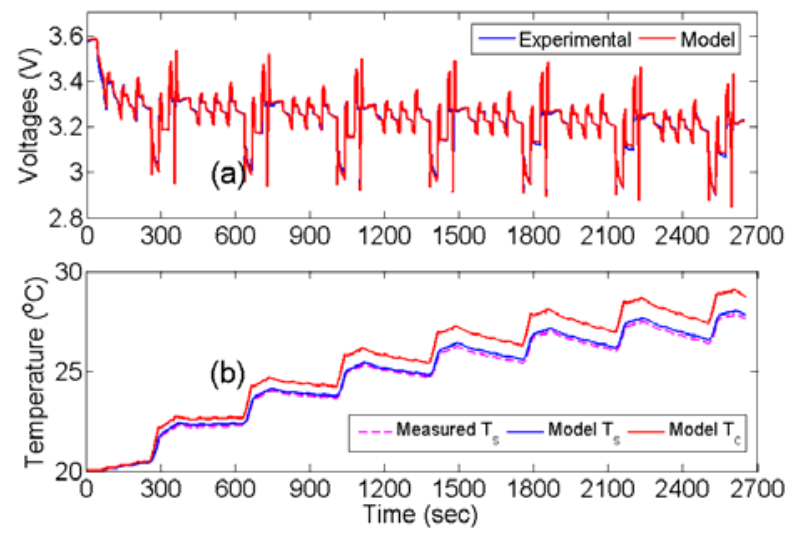

Fig. 13. Voltage and temperature variations under the DST cycles. (a) Experimental and model voltage; (b) Measured surface temperature, and core and surface temperatures of thermal model. 


\section{Diagnostic experimental evaluation}

The effectiveness of proposed FDI scheme is experimentally tested under the DST cycles. Due to the incorporated low pass filter into the measurement units, the large noise influences will be eliminated. The ambient temperature $T_{f}$ is controlled at $20^{\circ} \mathrm{C}$ in the thermal chamber.

The aim of the proposed diagnostic scheme is to identify the sensor fault in the early stage. In other words, the diagnostic scheme could identify the fault, even if the fault is very small. Taking the voltage sensor as an example, the upper voltage $(3.6 \mathrm{~V})$ corresponds to the full charge, i.e. SoC $100 \%$, while the lower voltage $(2.0 \mathrm{~V})$ means that there is no charge in the battery. Therefore, a very small voltage sensor fault would affect the operations of BMS, may resulting the battery suffering from over-charge or over-discharge.

The fault injection is in itself an art. The major considerations for the fault injection are as follows: (a) The injected fault cannot result in potential damage to the battery; (b) The injected fault cannot result in BMS shutting the operation. In other words, the measurements in the sensor faulty cases are acceptable by BMS. As specified in BMS during the experiments of fault injection, the maximum discharge current is $30 \mathrm{~A}$ (maximum continuous discharge current is 70A for A123 26650 specified by manufacturer [29]); the upper and lower voltage limit is $3.6 \mathrm{~V}$ and $2.6 \mathrm{~V}$; the maximum operating temperature is $55^{\circ} \mathrm{C}$ same as specified by manufacturer [29].

In MSO1, the initial states for EKF estimation are set as $x_{0}=\left[V_{1}, V_{2}, S o C\right]^{\mathrm{T}}=[0,0,1]^{\mathrm{T}}$, and the initial thermal model states $T_{c}$ and $T_{s}$ are the ambient temperature $T_{f}$. The initial error covariance $P_{0}$, noise covariance $C_{0} v_{-} w$ and Cov_v of EKF algorithm in MSO1 are set through trial-and-error test as follows,

$$
\begin{gathered}
P_{0}=\left[\begin{array}{ccc}
0.01 & 0 & 0 \\
0 & 0.01 & 0 \\
0 & 0 & 0.01
\end{array}\right] \\
C o v_{-} w=\left[\begin{array}{ccc}
0.001 & 0 & 0 \\
0 & 0.000001 & 0 \\
0 & 0 & 0.001
\end{array}\right] \\
\text { Cov_v }=1
\end{gathered}
$$

In MSO2, the initial value of model states are defined as $\left[V_{1}, V_{2}, S o C, T_{c}, T_{s}\right]^{\mathrm{T}}=[0,0,1,20,20]^{\mathrm{T}}$, and the parameter $\beta$ is set to 0.9 .

\subsection{Fault diagnosis without any faulty sensors}

The aim of this part is to investigate the residual response without any faulty sensor. Taking one experimental test as an example, the measured current, voltage, surface temperature, and estimated SoC in the sensor fault-free case are plotted in Fig. 14, while Fig. 15 shows the residuals $r_{1}$ and $r_{2}$, and CUSUM test results. It can be found from Fig 14 that all the sensor signals reside in the acceptable range of BMS, and the estimated SoC shows a good agreement with the experimental $\mathrm{SoC}$ as shown in Fig. 14 (d). 
It can be observed from Fig. 15 (a) and (b) that the residuals are non-zero, and show non-stationary behaviors. This is because of the existing modeling error and system noise. Through CUSUM test, the results $g\left(r_{1}\right)$ and $g\left(r_{2}\right)$ are plotted in Fig. 15 (c) and (d). The threshold $J_{1}$ and $J_{2}$ are calibrated to be 60 and 100 respectively. The alarm thresholds $J$ are calibrated through an extensive number of experimental tests. To avoid the false alarm, the thresholds are set to be slightly greater than the maximum magnitudes of $g(r)$ under the fault-free tests.

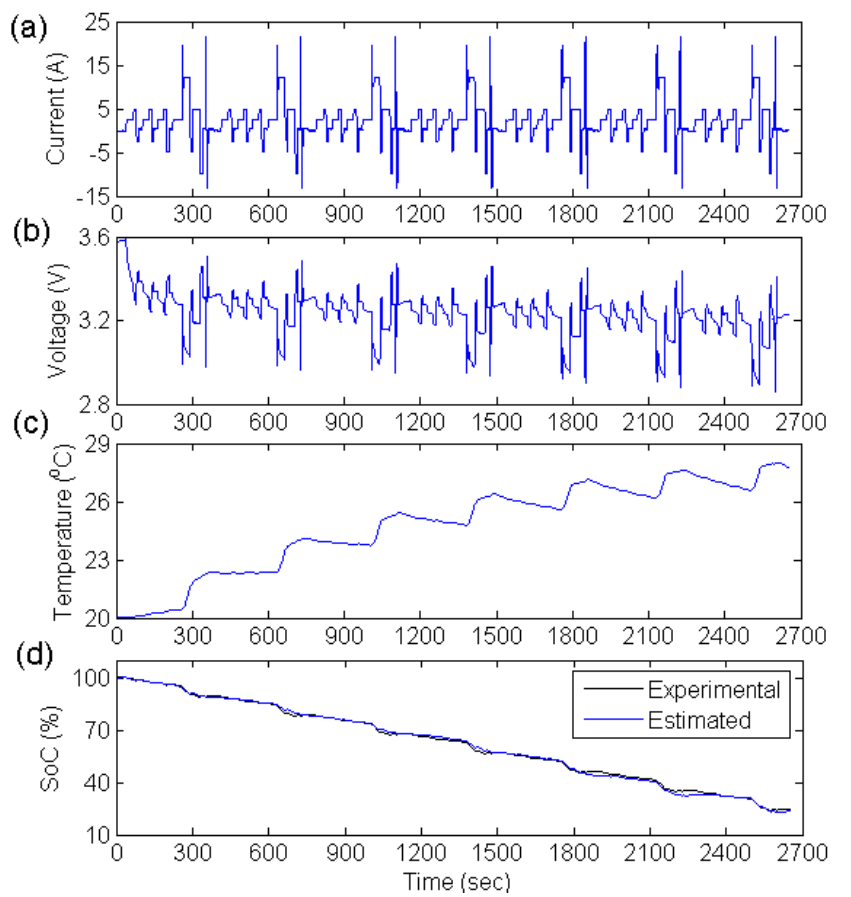

Fig. 14. Signals without any sensor fault. (a) Measured current; (b) Measured voltage; (c) Measured surface temperature; (d) Comparison between the experimental and estimated SoC.
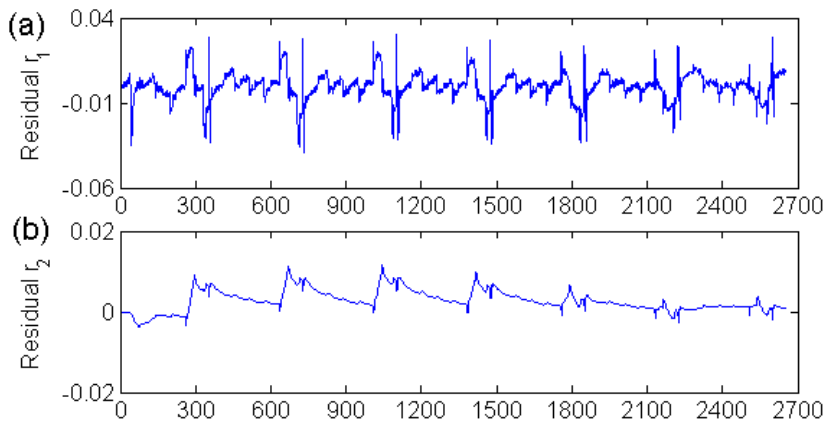

(c)
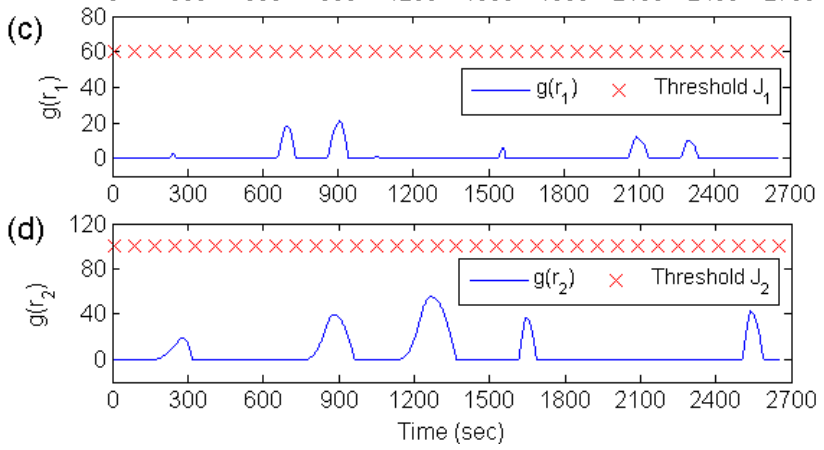
Fig. 15. Residuals and CUSUM test results in sensor fault-free case. (a) Residual $r_{1}$; (b) Residual $r_{2}$; (c) CUSUM test results $g\left(r_{1}\right)$; (d) CUSUM test results $g\left(r_{2}\right)$.

\subsection{Fault diagnosis with a current sensor fault}

Fig. 16 depicts the measured current, estimated SoC, residual $r_{1}$ and $r_{2}$ when the current sensor fault $f_{I}$ with $20 \%$ off scaling is abruptly injected at the time 1500 s. It can be concluded from Fig. 16 (b) that the SoC estimation will be affected in the current senor faulty case (Note that in the real time, the SoC is not known under the sensor fault-free condition), and the error is out of the acceptable range $5 \%$.

The magnitude of residual $r_{1}$ increases from the time 1500s as shown in Fig. 16 (c), but the behavior is similar before and after the fault injected. This is hard to tell from Fig. 16 (c) that a fault is occurred by using the constant thresholding approach. Through the CUSUM test, the diagnostic results are plotted in Fig. 17. It can be found from Fig. 17 (a) that $g\left(r_{1}\right)$ continually increases from the time $1565 \mathrm{~s}$, and at the time $1610 \mathrm{~s}$ the alarm threshold $J_{1}$ is exceeded as shown in the amplified portion of $g\left(r_{1}\right)$ in Fig. 17 (b), indicating the presence of a fault. Meanwhile, the $g\left(r_{2}\right)$ does not respond to this fault at all as shown in Fig. 17 (c). According to the residual characteristics in Table 2, the current sensor fault $f_{I}$ is identified as only residual $r_{1}$ responds to this fault. The isolated fault $f_{I}$ is plotted in Fig. 17 (d), and there is 110 s detection time delay. This may be attributed to the modeling error and the threshold value. The possible explanation for the threshold is that it may be a little high, but there may be a false alarm if the threshold is set lower. This tells that there is a tradeoff between the false alarm and detection time.
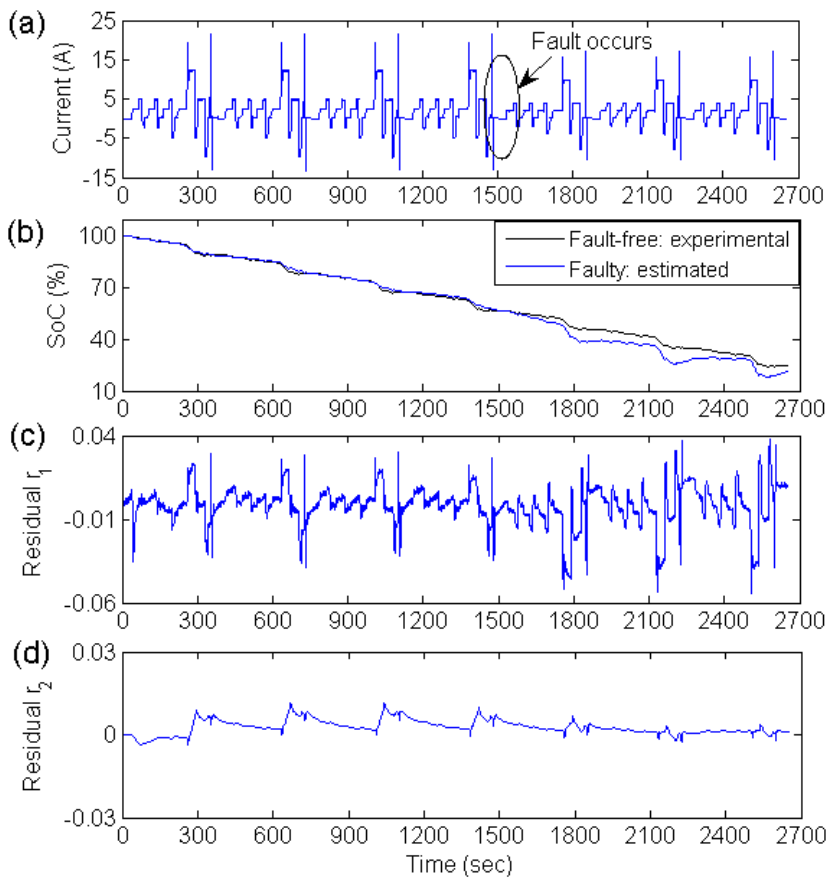

Fig. 16. Signals in the current sensor fault case. (a) Measured current; (b) Comparison between the experimental $\mathrm{SoC}$ in the sensor fault-free case, and estimated SoC in the sensor faulty case; (c) Residual $r_{1}$; (d) Residual $r_{2}$. 


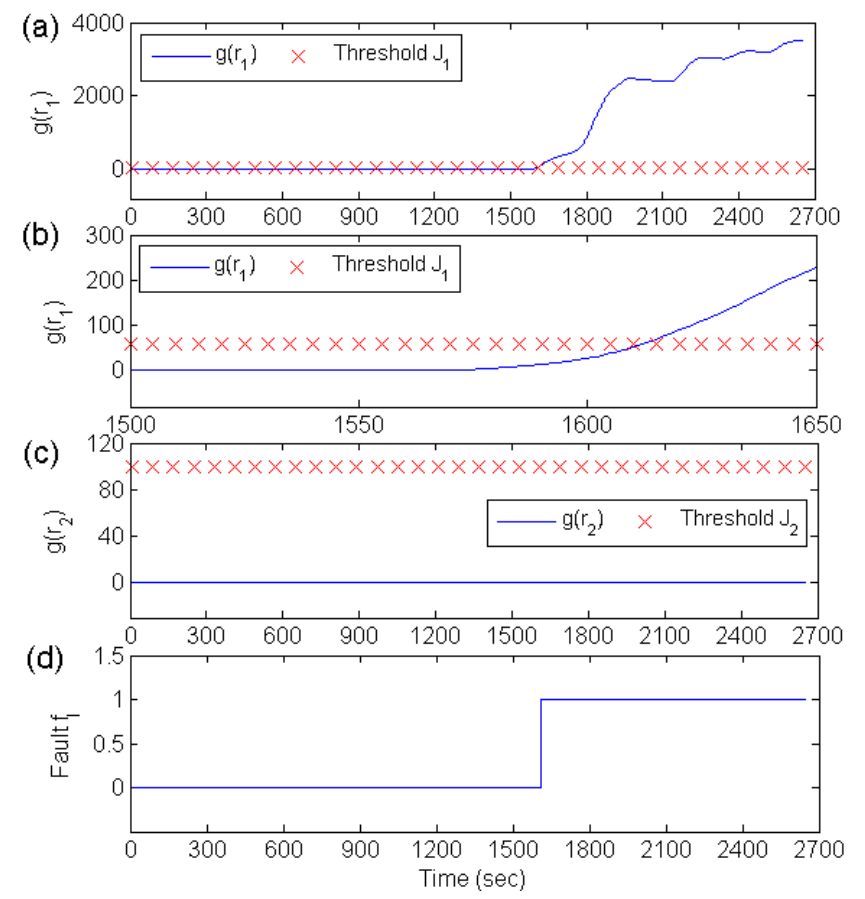

Fig. 17. Diagnostic results for the current sensor fault. (a) CUSUM test results $g\left(r_{1}\right)$; (b) Amplified portion of $g\left(r_{1}\right)$; (c) CUSUM test results $g\left(r_{2}\right)$; (d) Isolated current sensor fault $f_{I}$ signal.

\subsection{Fault diagnosis with a voltage sensor fault}

Fig. 18 plots the measured voltage, estimated SoC, residual $r_{1}$ and $r_{2}$ when the voltage sensor fault $f_{V t}$ with $0.03 \mathrm{~V}$ bias is abruptly injected at the time $1500 \mathrm{~s}$. Similarly, the SoC estimation will be affected in the voltage senor faulty case as shown in Fig. 18 (b). It can be found from Fig. 18 (c) and (d) that both residual $r_{1}$ and $r_{2}$ decrease after fault injected, but after the time 1800s the behavior of residual $r_{1}$ is similar with that before the fault injected. Through the CUSUM test, the diagnostic results are plotted in Fig. 19. It can be found from Fig. 19 (a) and (c) that $g\left(r_{1}\right)$ exceeds its threshold $J_{1}$ at the time $1529 \mathrm{~s}$, while the alarm threshold $J_{2}$ is crossed by $g\left(r_{2}\right)$ at the time $1760 \mathrm{~s}$. The amplified portions of $g\left(r_{1}\right)$ and $g\left(r_{2}\right)$ are shown in Fig. 19 (b) and (d) showing more details. It can be concluded from the response time of $g\left(r_{1}\right)$ and $g\left(r_{2}\right)$ to the fault $f_{V t}$ that residual $r_{1}$ is much more sensitive to this fault than residual $r_{2}$. This is reasonable as the residual $r_{1}$ is generated through the voltage signal, while the temperature dynamic is used for the generation of residual $r_{2}$. Finally, through matching the residual characteristics in Table 2, the fault $f_{V t}$ is isolated as both residuals respond to this fault, and the identified fault $f_{V t}$ signal is plotted in Fig. 19 (e). 

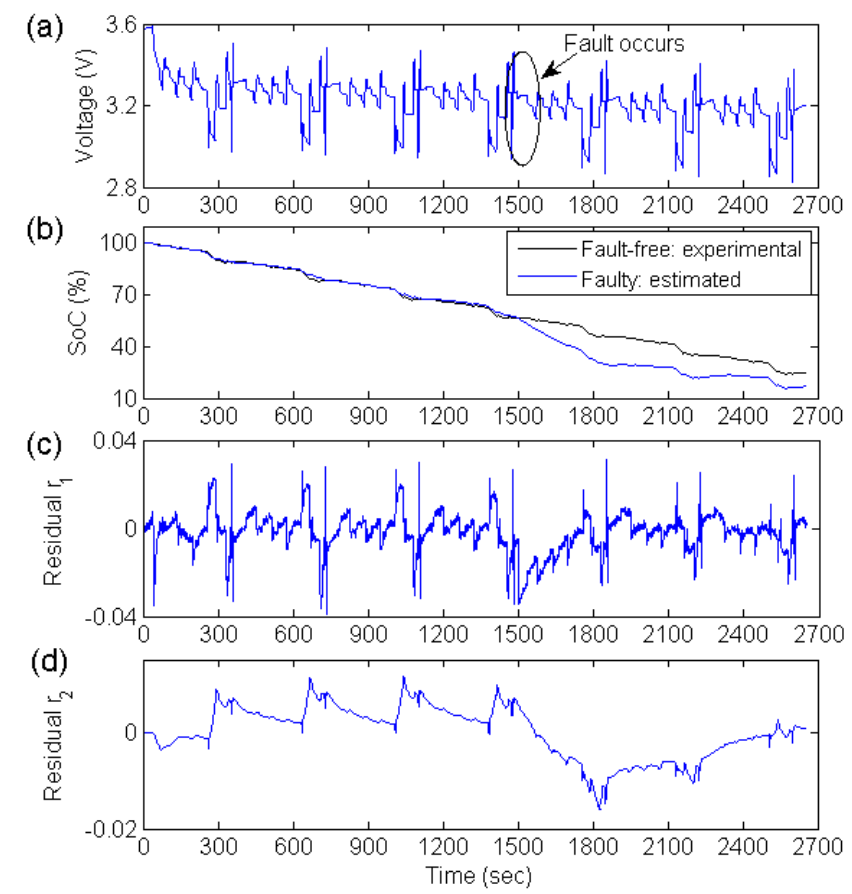

Fig. 18. Signals in the voltage sensor fault. (a) Measured voltage; (b) Comparison between the experimental SoC in the sensor fault-free case, and estimated $\mathrm{SoC}$ in the sensor faulty case; (c) Residual $r_{1}$; (d) Residual $r_{2}$.

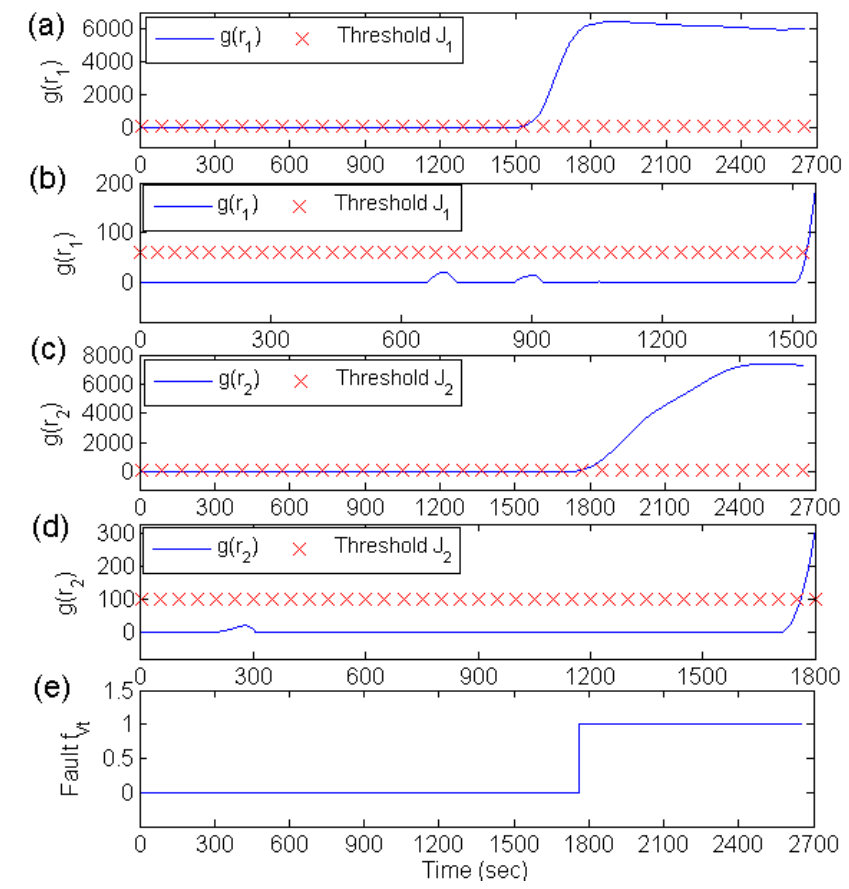

Fig. 19. Diagnostic results for the voltage sensor fault. (a) CUSUM test results $g\left(r_{1}\right)$; (b) Amplified portion of $g\left(r_{1}\right)$; (c) CUSUM test results $g\left(r_{2}\right)$; (d) Amplified portion of $g\left(r_{2}\right)$; (e) Isolated voltage sensor fault $f_{V t}$ signal.

\subsection{Fault diagnosis with a temperature sensor fault}

Fig. 20 depicts the measured surface temperature, residual $r_{1}$ and $r_{2}$ when the surface temperature sensor fault $f_{T S}$ with $0.8{ }^{\circ} \mathrm{C}$ bias is abruptly injected at the time $1500 \mathrm{~s}$. The thermal management system does not respond as the 
measured temperature resides in the specified operating range. It can be observed from Fig. 20 (b) and (c) that after the fault injected, the residual $r_{1}$ remains unchanged, while the residual $r_{2}$ has a dramatic increase and then quickly decreases. Through the CUSUM test, the diagnostic results are plotted in Fig. 21. Contrary to the current sensor fault scenario, only $g\left(r_{2}\right)$ responds to this fault, and at the time $1530 \mathrm{~s}$ the threshold $J_{2}$ is exceeded as shown in Fig. 21 (b) and (c). According to the residual characteristics in Table 2, the fault $f_{T s}$ is identified as only residual $r_{2}$ responds to this fault, and the isolated fault $f_{T S}$ is plotted in Fig. 21 (d).
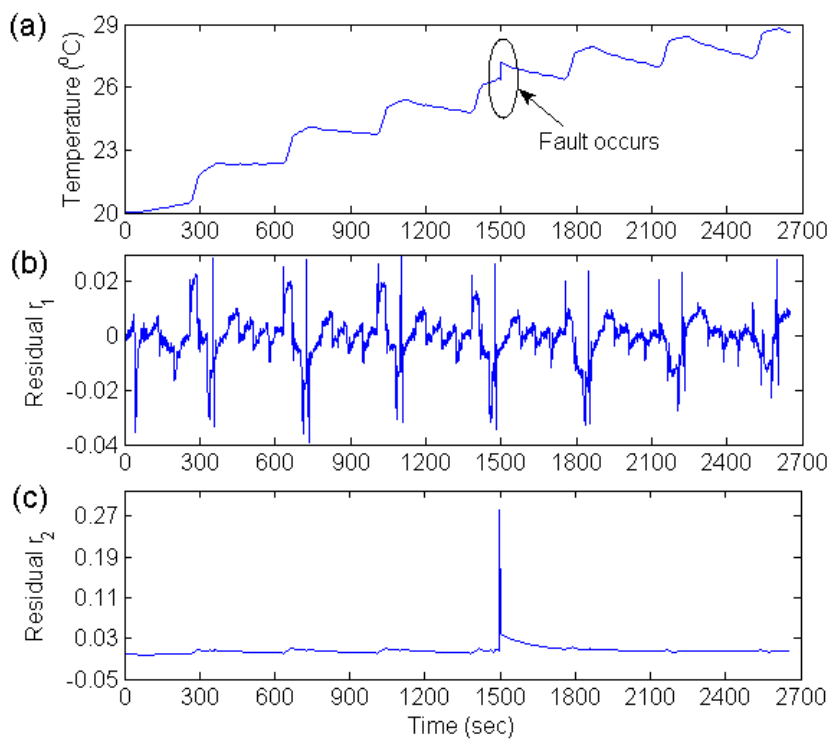

Fig. 20. Signals in the surface temperature sensor fault case. (a) Measured surface temperature; (b) Residual $r_{1}$; (c) Residual $r_{2}$.
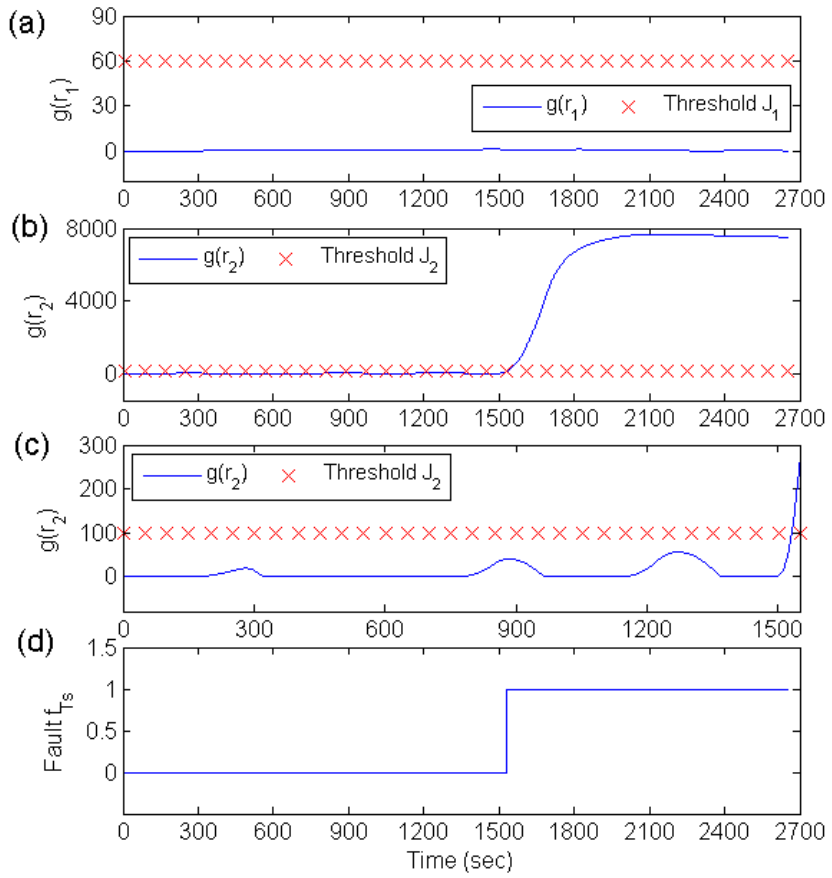

Fig. 21. Diagnostic results for the surface temperature sensor fault. (a) CUSUM test results $g\left(r_{1}\right)$; (b) CUSUM test results $g\left(r_{2}\right)$; (c) Amplified portion of $g\left(r_{2}\right)$; (d) Isolated surface temperature sensor fault $f_{T S}$ signal. 


\subsection{Fault diagnosis with different fault sizes, modes and injection times}

Different fault sizes, modes and injection times are considered for the current, voltage and surface temperature sensor. For the current sensor, the injected fault are set as $f_{I}=[ \pm 10 \% ; \pm 15 \% ; \pm 3 \mathrm{~A} ; \pm 5 \mathrm{~A}]$. For the voltage sensor, the injected fault are set as $f_{V t}=[ \pm 0.03 \mathrm{~V} ; \pm 0.05 \mathrm{~V} ; \pm 1 \% ; \pm 1.5 \%]$, while for the surface temperature sensor, the injected fault are set as $f_{T S}=\left[ \pm 1^{\circ} \mathrm{C} ; \pm 3^{\circ} \mathrm{C} ; \pm 5 \% ; \pm 10 \%\right]$. The fault injection time is random selected. To evaluate the effectiveness of the proposed FDI scheme, the following metrics are considered.

Isolation time (IT). Time from fault injected to first isolate the fault correctly.

False detection rate (FDR). The fraction of tests that the fault is detected in the no-fault case.

Missed isolation rate (MIR). The fraction of tests that does not achieve the accurate fault isolation. For instance, in total 10 experimental tests, there is only one test that cannot detect and isolate the injected fault, and the MIR would be 0.1 .

Through an extensive number of experimental tests, the maximum, minimum, and mean isolation time (IT), false detection rate (FDR), as well as the missed isolation rate (MIR) are summarized in Table 5. The isolation times are given in second.

\section{Table 5}

Summary of the IT, FDR and MIR for injected faults. All isolation times are specified in second.

\begin{tabular}{ccccc}
\hline \hline & & $f_{I}$ & $f_{V t}$ & $f_{T s}$ \\
\hline & Max & 267 & 314 & 50 \\
IT & Min & 16 & 96 & 10 \\
& Mean & 102.625 & 224.615 & 23.75 \\
FDR & & 0 & 0 & 0 \\
MIR & & 0 & 0 & 0 \\
\hline \hline
\end{tabular}

It can be concluded from Table 5 that there is no false detection and missed isolation. The maximum isolation time is $314 \mathrm{~s}$, which is for the voltage sensor fault $f_{V t}$ with $+0.03 \mathrm{~V}$ bias. But it is still acceptable in the real industry application. For each specific fault, the maximum and minimum isolation time correspond to the minimum and maximum fault size respectively. This tells that the isolation time is dependent on the fault size. Also, it should be noted that these metric values in Table 5 are correlated to the alarm threshold. If the threshold is selected too high, the fault isolation time would be longer, and maybe the fault cannot be detected.

\section{Conclusions}

This paper presents a systematic model-based FDI scheme for the lithium-ion battery to detect and isolate the current, voltage and temperature sensor fault. The proposed scheme is dependent on the sequential residual 
generation using structural analysis theory and statistical CUSUM test. Structural analysis pre-analyzes the sensor fault detectability and isolability without the accurate information of battery parameters. It also helps to identify the analytical redundancy part, i.e. structural over-determined part, by analyzing the structural model represented by an incidence matrix. Through extracting and selecting the minimally structural over-determined (MSO) sets, different diagnostic tests can be constructed.

To meet the battery sensors diagnostic requirement, only two MSO sets are needed and selected. EKF is used to address the issue of inaccurate initial SoC in MSO1. As for MSO2, an advanced technique is applied to avoid the derivation of battery temperature variable. The generated residuals from these two diagnostic tests are further evaluated by a statistical CUSUM test to determine the presence of fault. The effectiveness of the proposed FDI scheme is experimentally tested on a cylindrical lithium iron phosphate battery under realistic driving cycles, and different fault sizes, modes and injection times are considered. The results show the faults can be accurately identified in a reasonable time.

\section{Acknowledgement}

The authors would like to thank Prof. Erik Frisk and Mattias Krysander at Linköping University, for the substantial help on structural analysis theory. This work was supported by US Department of Energy under Award Number DE-PI0000012 in part, and National High Technology Research and Development Program of China (2012AA111603) in part.

\section{References}

[1] S. F. Tie and C. W. Tan, "A review of energy sources and energy management system in electric vehicles," Renew. Sustain. Energy Rev., vol. 20, pp. 82-102, Apr. 2013.

[2] L. Lu, X. Han, J. Li, J. Hua, and M. Ouyang, "A review on the key issues for lithium-ion battery management in electric vehicles," J. Power Sources, vol. 226, pp. 272-288, Mar. 2013.

[3] Y. Hu, S. Yurkovich, Y. Guezennec, and B. J. Yurkovich, "A technique for dynamic battery model identification in automotive applications using linear parameter varying structures," Control Eng. Pract., vol. 17, no. 10, pp. 1190-1201, Oct. 2009.

[4] G. L. Plett, "Extended Kalman filtering for battery management systems of LiPB-based HEV battery packs Part 3. State and parameter estimation," J. Power Sources, vol. 134, no. 2, pp. 277-292, 2004.

[5] X. Zhang, R. Grube, K. K. Shin, M. Salman, and R. S. Conell, "Parity-relation-based state-of-health monitoring of lead acid batteries for automotive applications," Control Eng. Pract., vol. 19, no. 6, pp. 555-563, 2011.

[6] H. Fang, Y. Wang, Z. Sahinoglu, T. Wada, and S. Hara, "State of charge estimation for lithium-ion batteries: An adaptive approach," Control Eng. Pract., vol. 25, no. 1, pp. 45-54, 2014.

[7] E. Balaban, A. Saxena, P. Bansal, K. F. Goebel, and S. Curran, "Modeling, detection, and disambiguation of sensor faults for aerospace applications," IEEE Sens. J., vol. 9, no. 12, pp. 1907-1917, 2009. 
[8] J. Marcicki, S. Onori, and G. Rizzoni, "Nonlinear fault detection and isolation for a lithium-ion battery management system," in Proceedings of 2010 ASME Dynamic Systems and Control Conference, 2010.

[9] C. Mikolajczak, M. Kahn, K. White, and R. T. Long, "Lithium-Ion Batteries Hazard and Use Assessment," Quincy, MA, 2011.

[10] W. Chen, W. Chen, M. Saif, M. Li, and H. Wu, "Simultaneous Fault Isolation and Estimation of Lithium-Ion Batteries via Synthesized Design of Luenberger and Learning Observers," IEEE Trans. Control Syst. Technol., vol. 22, no. 1, pp. 290-298, Jan. 2014.

[11] A. Sidhu, A. Izadian, and S. Anwar, "Adaptive Nonlinear Model-Based Fault Diagnosis of Li-Ion Batteries," vol. 62, no. 2, pp. 1002-1011, 2015.

[12] C. Svärd and M. Nyberg, "Automated design of an FDI system for the wind turbine benchmark," J. Control Sci. Eng., vol. 2012, 2012.

[13] C. Svärd, M. Nyberg, E. Frisk, and M. Krysander, "Automotive engine FDI by application of an automated model-based and data-driven design methodology," Control Eng. Pract., vol. 21, no. 4, pp. 455-472, 2013.

[14] X. Lin, H. E. Perez, S. Mohan, J. B. Siegel, A. G. Stefanopoulou, Y. Ding, and M. P. Castanier, “A lumpedparameter electro-thermal model for cylindrical batteries," J. Power Sources, vol. 257, pp. 1-11, 2014.

[15] X. Lin, H. E. Perez, J. B. Siegel, A. G. Stefanopoulou, Y. Li, R. D. Anderson, Y. Ding, and M. P. Castanier, "Online Parameterization of Lumped Thermal Dynamics in Cylindrical Lithium Ion Batteries for Core Temperature Estimation and Health Monitoring," IEEE Trans. Control Syst. Technol., vol. 21, no. 5, pp. 1745-1755, 2013.

[16] M. Blanke, M. Kinnaert, J. Lunze, and M. Staroswiecki, Diagnosis and Fault-Tolerant Control, 2nd Editio. Springer, 2006.

[17] M. Krysander, J. Åslund, and M. Nyberg, "An efficient algorithm for finding minimal overconstrained subsystems for model-based diagnosis," IEEE Trans. Syst. Man, Cybern. Part A Syst. Humans, vol. 38, no. 1, pp. 197-206, 2008.

[18] D. Düştegör, E. Frisk, V. Cocquempot, M. Krysander, and M. Staroswiecki, "Structural analysis of fault isolability in the DAMADICS benchmark," Control Eng. Pract., vol. 14, no. 6, pp. 597-608, 2006.

[19] M. Krysander and E. Frisk, "Sensor Placement for Fault Diagnosis," IEEE Trans. Syst. Man, Cybern. Part A Syst. Humans, vol. 38, no. 6, pp. 1398-1410, 2008.

[20] C. Sundstr, E. Frisk, and L. Nielsen, "Selecting and Utilizing Sequential Residual Generators in FDI Applied to Hybrid Vehicles," IEEE Trans. Syst. Man, Cybern. Part ASystems Humans, vol. 44, no. 2, pp. 172-185, 2014.

[21] C. Svärd, "Methods for Automated Design of Fault Detection and Isolation Systems with Automotive Applications," Linköping University, 2012.

[22] C. Svärd and M. Nyberg, "Residual Generators for Fault Diagnosis Using Computation Sequences With Mixed Causality Applied to Automotive Systems," vol. 40, no. 6, pp. 1310-1328, 2010. 
[23] C. Svärd, M. Nyberg, and E. Frisk, "A Greedy Approach for Selection of Residual Generators," in The 22nd Internaltional Workshop on Priciples of Diagnosis, 2011, pp. 1-8.

[24] M. Dubarry, V. Svoboda, R. Hwu, and B. Y. Liaw, "Capacity loss in rechargeable lithium cells during cycle life testing: The importance of determining state-of-charge,” J. Power Sources, vol. 174, no. 2, pp. 1121-1125, Dec. 2007.

[25] D. Simon, Optimal State Estimation Kalman, H Infinite, and Nonlinear Approaches. Wiley-Interscience, 2006.

[26] G. Rizzoni. "Lecture 14: Statistical inference methods for residual analysis and change detection," University Lecture, 2013

[27] B. Schweighofer, K. M. Raab, and G. Brasseur, "Modeling of High Power Automotive Batteries by the Use of an Automated Test System,” IEEE Trans. Instrum. Meas., vol. 52, no. 4, pp. 1087-1091, 2003.

[28] I.N.L., "Battery Test Manual for Plug-In Hybrid Electric Vehicles,” 2010.

[29] I. A123 Systems, “Nanophosphate ® High Power Lithium Ion Cell ANR26650M1-B,” 2011. 